\title{
Scal atrial natriuretic peptide gene polymorphisms and their possible association with postoperative atrial fibrillation - a preliminary report
}

\author{
Janusz Siebert ${ }^{1}$, Łukasz Lewicki ${ }^{1}$, Jolanta Myśliwska², Monika Młotkowska ${ }^{3}$, Jan Rogowski ${ }^{4}$
}

\author{
${ }^{1}$ Department of Family Medicine, University Centre for Cardiology, Medical University \\ of Gdansk, Gdansk, Poland \\ 2Department of Immunology and Histology, Medical University of Gdansk, Gdansk, \\ Poland \\ ${ }^{3}$ Department of Molecular Medicine, Medical University of Gdansk, Gdansk, Poland \\ ${ }^{4}$ Department of Cardiosurgery, Medical University of Gdansk, Gdansk, Poland
}

Submitted: 1 April 2015

Accepted: 29 June 2015

Arch Med Sci 2017; 13, 3: 568-574

DOI: 10.5114/aoms.2016.58270

Copyright @ 2016 Termedia \& Banach

\section{Abstract}

Introduction: Atrial fibrillation (AF) is a frequently encountered complication after coronary artery bypass grafting (CABG), but its underlying mechanisms are still unclear. The natriuretic peptides have been reported as markers for predicting the occurrence of postoperative AF. This study evaluates whether the Scal ANP gene polymorphisms predict the occurrence of postoperative AF. Material and methods: A prospective study of 203 consecutive patients with coronary artery disease undergoing elective CABG was undertaken for atrial natriuretic peptide (ANP) Scal gene polymorphism. Several perioperative data were analysed. Postoperative AF was defined as lasting for at least 15 min, confirmed by 12 -lead ECG and occurring within 6 postoperative days. The Scal polymorphism of the ANP gene was determined by polymerase chain reaction (PCR). Size-dependent separation of the PCR products on a polyacrylamide gel was followed by staining with ethidium bromide.

Results: The total frequency of AF was $19.7 \%$. The frequencies of Scal ANP gene polymorphisms were as follows: A1A1 4.90\%, A1A2 $59.60 \%$ and A2A2 $35.46 \%$. In order to assess the hypothesis that the A2 allele is a marker of increased risk of postoperative atrial fibrillation, the odds ratio (OR) was calculated: $A 2$ vs. non- $A 2, O R=0.98(0.23-4.1), p=0.97$, which was not significant. The odds ratios for $A 2 A 2$ and $A 1 A 1$ were not significant either: $A 2 A 2$ vs. non-A2A2, $O R=1.11(0.54-2.29), p=0.76$, and $A 1 A 1$ vs. non-A1A1, $\mathrm{OR}=1.17(0.23-5.92), p=0.84$.

Conclusions: ANP genotype did not predispose to the incidence of "new-onset" AF.

Key words: coronary artery disease, gene polymorphisms, coronary artery bypass grafting, Scal ANP, atrial fibrillation.

\section{Introduction}

Atrial fibrillation (AF) is a common complication after coronary artery bypass grafting (CABG) [1-3]. Arrhythmia is considered to be one reason for increased peri-operative morbidity, prolonged hospital stay and increased costs $[1,4]$. The pathogenesis of postoperative AF is still not well established. The best documented risk factors are increased age, congestive heart failure and peri-operative ischaemia [1, 2, 4-6]. Studies suggest

\author{
Corresponding author: \\ Łukasz Lewicki MD, PhD \\ University Centre for \\ Cardiology \\ Medical University of Gdansk \\ 2 Dębinki St \\ 80-210 Gdansk, Poland \\ Phone: +48 5834915 75, \\ +48501702885 \\ Fax: +48 583491576 \\ E-mail: luklewicki@gmail.com
}


that an important role is played by a peri-operatively induced systemic inflammatory response in the pathogenesis of AF [7]. The recently published data showed that perioperative treatment with atorvastatin is useful to decrease the incidence of AF in patients undergoing isolated heart valve surgery [8]. Moreover, a genetic predisposition to postoperative AF has been proved [9]. Among various pathophysiological factors predisposing to development of paroxysmal AF, interesting ones seem to be increased P-wave duration and dispersion that are observed in patients with diabetic autonomic neuropathy [10]. More recently, a simple risk score for prediction of postoperative AF has been validated [11]. In this large observational study several clinical features, i.e. age, chronic obstructive pulmonary disease, need of preoperative intra-aortic balloon pump, left ventricular ejection fraction less than $30 \%$, renal failure and any valve surgery were identified as independent predictors of postoperative AF. Although there are many well-identified clinical and biochemical risk factors, there is still a lack of sufficient data on genetic predictors of postoperative arrhythmia.

It has been demonstrated that atrial natriuretic peptide (ANP) is a hormone which plays an important role in congestive heart failure (CHF) and hypertension [12, 13]. The ANP has natriuretic, diuretic and vasodilatative properties. Increased atrial-wall tension as a result of increased intravascular volume stimulates release of ANP [14]. This phenomenon occurs in patients with heart failure but also during cardiac surgery. There is strong evidence that low-dose continuous infusion of human ANP during cardiac surgery leads to a reduction in central venous pressure, the systemic vascular resistance index and the pulmonary vascular resistance index. Additionally, a reduction in levels of renin, angiotensin-II, aldosterone, and pleural effusion has been observed in comparison with patients who did not receive ANP infusion [15]. As pleural effusion and fluid overload of the atrium are considered to be partially responsible for postoperative atrial fibrillation, these observations may suggest the potential role of ANP in the pathogenesis of the arrhythmia. On the other hand, a relationship between ANP gene polymorphism and a history of supraventricular tachycardia has been observed in patients with dilated cardiomyopathy [16].

The aim of the study was to explore whether the A2 allele is a marker of increased risk of postoperative atrial fibrillation.

\section{Material and methods}

\section{Study population}

The 203 white patients included in the study, 152 male and 51 female, were qualified for elec- tive coronary artery surgery. Written informed consent was obtained from each person. All consecutive patients were inhabitants of the Pomeranian region and were operated on at the Department of Cardiac Surgery at the Medical University of Gdansk. All the patients were interviewed by one physician before the operation and data were recorded on the following: angina status according to the Canadian Cardiovascular Society (CCS), history of myocardial infarction, arterial hypertension, diabetes and previous arrhythmias, including chronic and paroxysmal AF. Patients who had either persistent or permanent $A F$, atrial flutter or atrial tachycardia on a standard 12-lead ECG obtained on the day of the operation were excluded from the study. Twenty-four of the included patients had reduced ejection fraction heart failure (REF-HF) and 5 of them had EF less than $30 \%$. Ninety-five patients were operated on with standard CABG using extracorporeal circulation and 108 patients underwent off-pump coronary artery bypass surgery (OPCABG) without cardiopulmonary bypass, on a beating heart. The risk of peri-operative mortality was estimated according to EuroSCORE [17]. The study protocol was approved by the local ethics committee.

\section{Genetic analysis}

Genomic DNA was isolated from whole blood taken from a patient on the day before surgery using the Blood DNA Prep Plus spin-column system according to the protocol provided by the manufacturer (A\&A Biotechnology, Gdansk, Poland). The Scal site polymorphism of the ANP gene was determined by polymerase chain reaction (PCR) by standard techniques [18]. A fragment of 234 base pairs (bp) was amplified in the region encompassing exon 3 of the ANP gene, with the sense primer (5'-GGT GGG AAG CAG GTG GTC AGT ACT CAA GTT CAG AGG ATG GGC-3') and antisense primer (5'-CAC AAC TCC ATG GCA ACA AGA TGA CAC AAA TGC-3'). To detect $\mathrm{T} 2238 \rightarrow C$ transition, restriction enzyme digestion with Scal was performed according to the protocol provided by the manufacturer (Promega, Madison, Wis). The size-dependent separation of the PCR products on a $12 \%$ polyacrylamide gel was followed by staining with ethidium bromide. In the presence of the polymorphic site for the A2 allele 3 fragments were generated corresponding to sizes 117,96 and $21 \mathrm{bp}$. For the $\mathrm{A} 1$ allele a fragment of $234 \mathrm{bp}$ was split into 213 and 21 bp products [19], as shown in Figure 1.

\section{Arrhythmia analysis}

Atrial fibrillation was assessed using a continuous ECG monitor system (Hewlett Packard, USA) with the possibility of rhythm disturbance analy- 


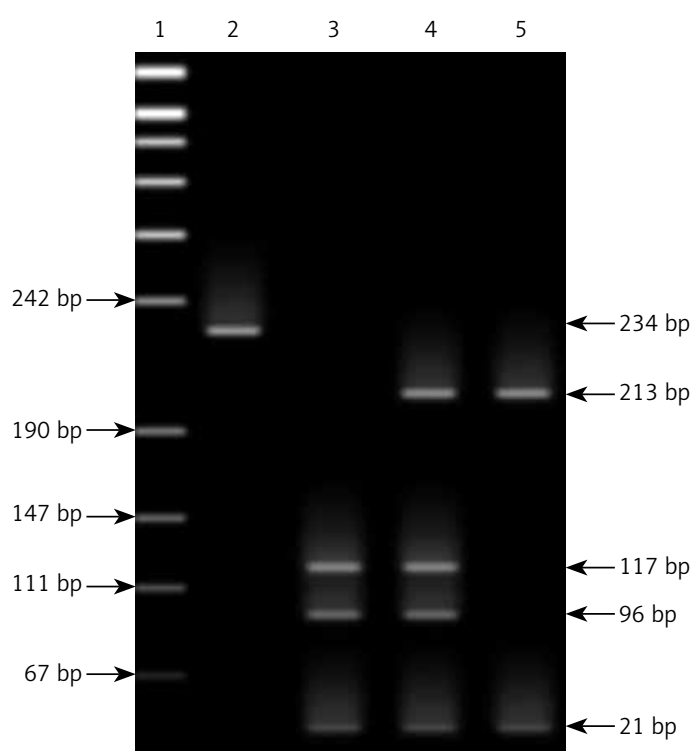

Figure 1. Genotyping by polyacrylamide gel electrophoresis of PCR-RFLP. A fragment of the ANP gene was amplified and products were digested with Scal restriction enzyme, subsequently run on $12 \%$ polyacrylamide gel using $1 \%$ solution ethidium bromide to visualize the fragments

1 - Marker, 2 - ANP amplified product, 3 - the homozygous A1 allele appeared as 213 and 21-bp bands, 4 - the homozygous A2 allele appeared as bands of 117 , 96 and 21 bp, 5 - the heterozygotes A1A2 exhibited all bands $(213,117,96$ and $21 \mathrm{bp})$.

Table I. Scal ANP genotype frequency in 203 patients with ischaemic heart disease admitted for CABG

\begin{tabular}{|c|c|}
\hline Scal ANP genotype & Number of patients (\%) \\
\hline $\mathrm{A} 2 \mathrm{~A} 2$ & $72(35.5)$ \\
\hline $\mathrm{A} 1 \mathrm{~A} 2$ & $121(59.6)$ \\
\hline $\mathrm{A} 1 \mathrm{~A} 1$ & $10(4.9)$ \\
\hline
\end{tabular}

sis during the patient's stay in the intensive care unit (ICU). Subsequently, during the first $24 \mathrm{~h}$ after leaving the ICU, each patient was monitored using the Space Lab system (Space Lab, USA). A standard 12-lead ECG was performed once a day and continually in any case of clinical manifestations of arrhythmia. The analysis took into account every incident of AF lasting longer than 15 min or requiring medical treatment because of the patient's instability. The period during which the patients were observed was restricted to 6 postoperative days.

\section{Statistical analysis}

Values are expressed as mean and standard deviations. The EuroSCORE data are median and range and were compared using the Kruskal-Wal- lis test. The $\chi^{2}$ test was used for analysis of the categorical variables, and Student's t-test was used for continuous variables with normal distribution. On the other hand, the Mann-Whitney $U$ test was used for non-normally distributed continuous variables. When more than two groups were compared, an ANOVA test was used. The relationship between Scal ANP gene variants and the incidence of "new-onset" AF was assessed and the odds ratios were calculated using a logistic regression model.

$P$-values of less than 0.05 were considered to be significant. Statistical analysis was performed using Statistica 10.0 (StatSoft).

\section{Results}

Allele distribution in this group of 203 patients is presented in Table I. There was dominance of A1A2 heterozygotes.

The clinical profile of the 203 patients with ischaemic heart disease admitted for CABG and OPCABG is presented according to Scal ANP genotype in Table II. In general, there were no significant differences between the patients' parameters. There were no significant differences in mean age, body mass index (BMI), left ventricle ejection fraction (LVEF) or left atrial size (LA) between the $A 2 A 2$ and $A 1 A 2$ groups. There was a significant difference in the length of time from the first angina pectoris episode to the time of cardiac surgery between patients with $\mathrm{A} 1 \mathrm{~A} 1$ and $\mathrm{A} 1 \mathrm{~A} 2,10.9$ years versus 8 years respectively. Patients with the A1A2 genotype had a higher level of plasma triglycerides compared to those with the A2A2 genotype, 185.7 and $150.7 \mathrm{mg} / \mathrm{dl}$ respectively.

Two hundred of the patients completed the 6 days follow-up. A total of 4 patients died peri-operatively, 2 with the $A 2 A 2$ and 2 with the $A 1 A 2$ genotype. Three deaths occurred in the first $24 \mathrm{~h}$ after surgery and 1 patient died on the $10^{\text {th }}$ postoperative day. All deaths were due to perioperative low output syndrome and none was caused directly by atrial fibrillation. A total of 40 (19.7\%) patients developed AF postoperatively. The distribution of AF in particular allele groups is presented in Figure 2.

ANP genotype did not predispose to the occurrence of "new-onset" AF, as shown in Table III.

The distribution between particular genotypes of other clinical data obtained during the intra- and postoperative periods is presented in Table IV. There were no significant differences in postoperative complications between any particular groups.

The limited number of the patients with the A1A1 genotype might have distorted our results. Therefore, as a next step, the patients carrying the $A 1 A 1$ and $A 1 A 2$ genotypes were analysed jointly to represent the A1-allele-positive participants, and 
Table II. Clinical profile of 203 patients with ischaemic heart disease admitted for CABG and OPCABG according to Scal ANP genotype. The analysis was made using the ANOVA test for continuous variables and $\chi^{2}$ test for categorical data

\begin{tabular}{|c|c|c|c|c|}
\hline \multirow[t]{2}{*}{ Parameter } & \multicolumn{3}{|c|}{ Scal ANP genotype } & \multirow[t]{2}{*}{$P$-value } \\
\hline & $\mathrm{A} 1 \mathrm{~A} 2$ & $\mathrm{~A} 2 \mathrm{~A} 2$ & $\mathrm{~A} 1 \mathrm{~A} 1$ & \\
\hline Number of patients & 121 & 72 & 10 & \\
\hline Age [years] & $62 \pm 9$ & $64 \pm 8.9$ & $63 \pm 7.19$ & 0.7 \\
\hline BMI & $27.9 \pm 4.1$ & $25.7 \pm 3.9$ & $28 \pm 2.9$ & 0.5 \\
\hline EuroSCORE & $3(0 ; 9)^{\star}$ & $3(0 ; 10)^{*}$ & $4(0 ; 6)^{\star}$ & 0.6 \\
\hline Duration of angina [years] & $8 \pm 7.5^{\star *}$ & $8.8 \pm 7.4$ & $10.9 \pm 9.7^{\star \star}$ & $0.004(0.4)$ \\
\hline NYHA & $1 \pm 0.73$ & $1 \pm 0.76$ & $1 \pm 0.69$ & 0.8 \\
\hline Total cholesterol [mg\%] & $225.9 \pm 51.2$ & $214 \pm 48$ & $216 \pm 51$ & 0.9 \\
\hline LDL cholesterol [mg\%] & $140.9 \pm 43.3$ & $132 \pm 41$ & $144 \pm 57.8$ & 0.5 \\
\hline HDL cholesterol [mg\%] & $43.2 \pm 12.5$ & $42 \pm 12$ & $45 \pm 13$ & 0.9 \\
\hline Triglycerides [mg\%] & $185.7 \pm 138.4^{\star *}$ & $150.7 \pm 66.4^{\star *}$ & $136 \pm 56$ & $0.04(0.1)$ \\
\hline Creatinine [mg\%] & $1.14 \pm 0.2$ & $1.1 \pm 0.2$ & $1.19 \pm 0.2$ & 0.7 \\
\hline EF & $53.6 \pm 10.2$ & $52.8 \pm 11.3$ & $47.5 \pm 9.5$ & 0.9 \\
\hline LA & $4 \pm 0.5$ & $4 \pm 0.5$ & $4.15 \pm 0.4$ & 0.6 \\
\hline LVESd & $3.5 \pm 0.8$ & $3.6 \pm 0.7$ & $4.1 \pm 1.2$ & 0.3 \\
\hline LVEDd & $5.2 \pm 0.8$ & $5.2 \pm 0.6$ & $5.6 \pm 0.8$ & 0.9 \\
\hline Male/female & $87 / 34$ & $55 / 17$ & $10 / 0$ & 0.49 \\
\hline Myocardial infarction history & 81 & 47 & 8 & 0.81 \\
\hline Diabetes & 32 & 16 & 1 & 0.49 \\
\hline Hypertension & 82 & 48 & 4 & 0.9 \\
\hline Paroxysmal atrial fibrillation & 8 & 3 & 0 & 0.7 \\
\hline
\end{tabular}

*Values are expressed as median and range and were compared using the Kruskal-Wallis test, ${ }^{* *} p<0.05$. CABG - coronary artery bypass grafting, OPCABG - off-pump coronary artery bypass grafting, LA - left atrial diameter, LVESd - left ventricle systolic diameter, LVEDd - left ventricle end-diastolic diameter, $A N P$ - atrial natriuretic peptide, $B M I$ - body mass index, EF-ejection fraction.

these were contrasted with the A2A2 genotype patients.

From the $3^{\text {rd }}$ to the $6^{\text {th }}$ day after surgery the frequency of the AF episodes decreased and the frequency of AF was not noticeably higher among the A2A2 patients $\left(\chi^{2} ; p=0.1\right)$.

A comparison of the distribution of patients according to NYHA grade revealed that the patients with the A2A2 genotype were not significantly shifted towards the higher NYHA values in relation to those with $\mathrm{A} 1 \mathrm{~A} 2$ and $\mathrm{A} 1 \mathrm{~A} 1$.

\section{Discussion}

According to our knowledge, this study is the first to test an association between Scal ANP gene polymorphism with the clinical peri-operative state in patients undergoing cardiac surgery. A previous study performed by a research group from our university showed that the A2A2 allele is associated with a higher incidence of histories of non-fatal myocardial infarction and more extensive CAD [20]. More recently Rubattu et al. demon-

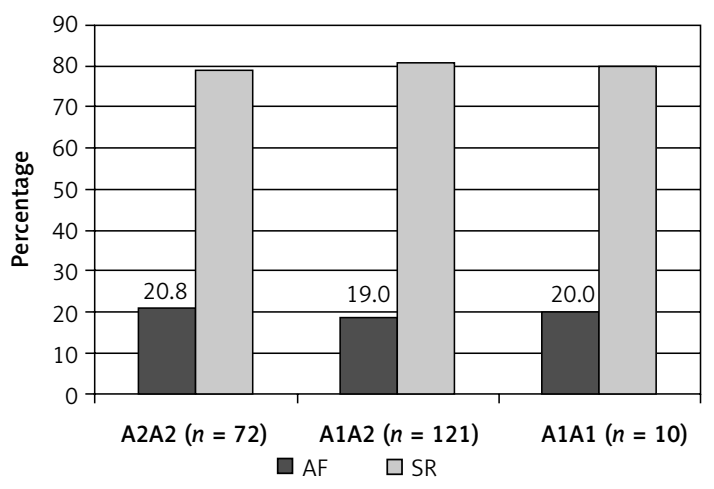

Figure 2. Incidence of atrial fibrillation after 203 CABG procedures

AF-atrial fibrillation, $S R$ - sinus rhythm, CABG - coronary artery bypass grafting. 
Table III. Relationship between Scal ANP gene variants and the incidence of "new-onset" AF in 192 patients (after excluding patients with a history of paroxysmal AF before the operation). The analysis was made using a logistic regression model

\begin{tabular}{|lcc|}
\hline OR for "new-onset" atrial fibrillation A1A2 vs. A2A2 and A1A1 & OR $=0.65(0.12-3.34)$ & $p=0.6$ \\
\hline OR for "new-onset" atrial fibrillation A2A2 vs. non A2A2 & OR $=1.35(0.59-3.07)$ & $p=0.46$ \\
\hline OR for "new-onset" atrial fibrillation A1A1 vs. non A1A1 & OR $=1.8(0.34-9.5)$ & $p=0.48$ \\
\hline
\end{tabular}

$A F-$ atrial fibrillation, ANP - atrial natriuretic peptide.

Table IV. Intra- and postoperative data in 203 CABG patients according the Scal ANP genotype. The analysis was made using the ANOVA test for continuous variables and $\chi^{2}$ test for categorical data

\begin{tabular}{|c|c|c|c|c|}
\hline \multirow[t]{2}{*}{ Parameter } & \multicolumn{3}{|c|}{ Scal ANP genotype } & \multirow[t]{2}{*}{$P$-value } \\
\hline & $\mathrm{A} 1 \mathrm{~A} 2$ & $\mathrm{~A} 2 \mathrm{~A} 2$ & $\mathrm{~A} 1 \mathrm{~A} 1$ & \\
\hline Number of patients & 121 & 72 & 10 & \\
\hline Neuro-mental complications & $5(4.1 \%)$ & $3(4.1 \%)$ & 0 & 0.7 \\
\hline Excessive bleeding & $7(5.8 \%)$ & $2(2.7 \%)$ & 0 & 0.4 \\
\hline Peri-operative death & $2(1.6 \%)$ & $2(2.7 \%)$ & 0 & 0.9 \\
\hline CABG/OPCABG & $59 / 62$ & $29 / 43$ & $7 / 3$ & 0.2 \\
\hline $1^{\text {st }}$ day drainage $[\mathrm{ml}]$ & $562 \pm 300.5$ & $554 \pm 258$ & $586.25 \pm 269.2$ & 0.8 \\
\hline CPK-MB & $33.2 \pm 86.7$ & $21 \pm 35.3$ & $15.6 \pm 16.9$ & 0.7 \\
\hline CPB time $[\mathrm{min}]$ & $90.47 \pm 31.5$ & $92.11 \pm 35.4$ & $71.5 \pm 17.7$ & 0.4 \\
\hline Duration of aortic cross clamp [min] & $47.5 \pm 18.6$ & $50 \pm 24$ & $39.8 \pm 11.3$ & 0.9 \\
\hline Number of distal anastomoses & $2.63 \pm 0.9$ & $2.69 \pm 0.9$ & $3.1 \pm 0.9$ & 0.2 \\
\hline
\end{tabular}

$C A B G$ - coronary artery bypass grafting, OPCABG - off-pump coronary artery bypass grafting, ANP - atrial natriuretic peptide, CPB cardiopulmonary bypass.

strated that ANP polymorphism is associated with higher risk for an acute coronary syndrome recurrence in patients with CAD [21]. Moreover, a recently published meta-analysis indicated that ANP $2238 \mathrm{~T} / \mathrm{C}$ polymorphism is related to increased risk of ischaemic stroke [22].

The interesting observation from our study is the distribution of the Scal ANP genotype and allele frequency in the study group. We observed a relatively large group of the A2A2 genotype (35.5\% and $27.5 \%$ respectively) in comparison with a previous study [20]. This may be a reflection of a selection bias in the population that included only patients undergoing CABG.

An elevated level of ANP was tested with respect to AF prediction after CABG in two other studies, which, however, did not show a significant correlation with postoperative arrhythmia [23, 24].

Loke et al. pointed out that in the healthy population, plasma levels of N-ANP, brain natriuretic peptide (BNP) and N-BNP were variably influenced by clinical covariates [25].

While all 3 peptides were higher in women, only $\mathrm{N}-\mathrm{ANP}$ and N-BNP were influenced by age. Levels of all peptides were inversely correlated with heart rate. When an immunoluminometric assay is used, plasma BNP is not influenced by age, in contrast to N-ANP and N-BNP. In constructing normal ranges for diagnostic use, covariates such as age and gender must be considered, in addition to the format of the assay being used [25]. Histological examination revealed that the collagen volume in the left atrial tissue was higher in AF than it was in SR and inversely correlated with plasma ANP. In addition, the messenger RNA expression of ANP and collagen types I and III was lower in AF than in sinus rhythm [26]. The spectrum of histological alterations is not completely known. Patients with long-standing AF and rheumatic heart disease had a very high prevalence of atrial amyloidosis. Amyloid deposition was more frequent in the left atrial appendage than in the right and correlates with AF duration and female gender. Amyloid deposition could constitute an additional histological feature in the structural remodelling of the atria during long-standing AF [27].

Atrial fibrillation was described as an independent determinant of higher N-ANP levels, and its association with LV dysfunction is blurred. Conversely, BNP was not independently associated with AF and was strongly determined by LV dysfunction, for which it was an independent marker [28]. Chello et al. suggested that plasma levels of ANP and BNP might be used in routine clinical 
practice to support echocardiography in detecting recovery of LV function after coronary surgery [29].

As the hormonal environment is known to be very unstable during the early postoperative period, we hypothesised that analysis of the pre-operative ANP genotype, which is constant, may support a new predictor of postoperative AF. In our study the overall incidence of AF after cardiac surgery was comparable in the $A 2 A 2$ and $A 1 A 2$ groups. On the other hand, the B-type natriuretic peptide has been reported as predicting the occurrence of postoperative AF. A pre-operatively elevated plasma BNP level was a strong and independent predictor of postoperative AF. This finding has important implications for identifying patients at higher risk of postoperative AF who could be considered for prophylactic anti-arrhythmic or $\beta$-blocker therapy [30]. On the other hand, Masson et al., in their more recently published study, found that both pre- and postoperative plasma levels of NT-proBNP were not independently associated with postoperative AF [31].

In conclusion, the obvious limitation of our study is the lack of statistical significance, which may be due to the relatively small population. Further analysis is required, especially in order to assess the incidence of postoperative AF in patients with the A1A1 genotype. The small number of patients with that genotype in our study group suggests that the $A 2$ allele is particularly expressed in patients with coronary artery disease. There is no evidence of an association between the genotype and new onset AF.

\section{Conflict of interest}

The authors declare no conflict of interest.

\section{References}

1. Aranki SF, Shaw DP, Adams DH, et al. Predictors of atrial fibrillation after coronary artery surgery. Current trends and impact on the hospital resources. Circulation 1996; 94: 390-7.

2. Creswell LL, Schuessler RB, Rosenbloom M, Cox JL. Hazards of postoperative atrial arrhythmias. Ann Thorac Surg 1993; 56: 539-49.

3. Mathew JP, Parks R, Savino JS, Friedman AS, Koch C, Mangano DT. Atrial fibrillation following coronary artery bypass graft surgery: predictors, outcomes, and resource utilization. MultiCenter Study of Perioperative Ischemia Research Group. JAMA 1996; 276: 300-6.

4. Almassi GH, Schowalter T, Nikolosi AC, et al. Atrial fibrillation after cardiac surgery - a major morbid event? Ann Surg 1997; 226: 501-13.

5. Al-Shanafey S, Dodds L, Langille D, Ali I, Henteleff $\mathrm{H}$, Dobson R. Nodal vessels disease as a risk factor for atrial fibrillation after coronary artery bypass graft surgery. Eur J Cardiothorac Surg 2001; 19: 821-826.

6. Kolvekar S, D’Souza A, Akhtar P, Reek C, Garratt C, Spyt T. Role of atrial ischaemia in development in atrial fibrillation following coronary artery bypass surgery. Eur J Cardiothorac Surg 1997; 11: 70-5.
7. Bruins P, te Velthuis H, Yazdanbakhsh AP, et al. Activation of the complement system during and after cardiopulmonary bypass surgery. Circulation 1997; 96: 3542-8.

8. Dehghani MR, Kasianzadeh M, Rezaei Y, Sepehrvand N. Atorvastatin reduces the incidence of postoperative atrial fibrillation in statin-naive patients undergoing isolated heart valve surgery: a double-blind, placebocontrolled randomized trial. J Cardiovasc Pharmacol Ther 2015; 20: 465-72.

9. Gaudino M, Andreotti F, Zamparelli R, et al. The -174G/C interleukin-6 polymorphism influences postoperative interleukin- 6 levels and postoperative atrial fibrillation. Is atrial fibrillation an inflammatory complication? Circulation 2003; 108 (Suppl. II): II-195- 9.

10. Bissinger A, Grycewicz T, Grabowicz W, Lubinski A. The effect of diabetic autonomic neuropathy on P-wave duration, dispersion and atrial fibrillation. Arch Med Sci 2011; 7: 806-12.

11. Mariscalco G, Biancari F, Zanobini M, et al. Bedside tool for predicting the risk of postoperative atrial fibrillation after cardiac surgery: the POAF score. J Am Heart Assoc 2014; 3: e000752.

12. Levin E, Gardner D, Samson W, et al. Natriuretic peptides. N Engl J Med 1998; 339: 321-8.

13. Kato N, Sugiyama T, Morita H, et al. Genetic analysis of the atrial natriuretic peptide gene in essential hypertension. Clin Sci 2000; 98: 251-8.

14. Lang RE, Tholken H, Ganten D, et al. Atrial natriuretic factor-circulating hormone stimulated by volume loading. Nature 1985; 314: 2642-7.

15. Sezai A, Shiono M, Orime Y, et al. Low-dose continuous infusion of human atrial natriuretic peptide during and after cardiac surgery. Ann Thorac Surg 2000; 69: 732-8.

16. Richter D, Soccio M, Needham E, et al. Investigation of the atrial natriuretic peptide gene in patients with idiopathic dilated cardiomyopathy. Eur Heart J 1997; 18 (Suppl.): 911 [abstract].

17. Roques F, Nashef SAM, Michel P, et al. Risk factors and outcome in European cardiac surgery: analysis of the EuroSCORE multinational database of 19030 patients. Eur J Cardiothorac Surg 1999; 15: 816-23.

18. Ramasawmy R, Kotea N, Lu C, et al. Investigation of the polymorphic Scal site by a PCR-based assay at the human natriuretic peptides (hANP) gene locus. Hum Genet 1992; 90: 323-4.

19. Nannipieri M, Manganiello M, Pezzatini A, et al. Polymorphism in the hANP (Human Atrial Natriuretic Peptide) gene, albuminuraia, and hypertension. Hypertension 2001; 6: 1416-22.

20. Gruchała M, Ciećwierz D, Wasag B, et al. Association of the Scal atrial natriuretic peptide gene polymorphism with nonfatal myocardial infarction and extent of coronary artery disease. Am Heart J 2003; 145: 125-31.

21. Rubattu S, De Giusti M, Farcomeni A, et al. T2238C ANP gene variant and risk of recurrent acute coronary syndromes in an Italian cohort of ischemic heart disease patients. J Cardiovasc Med 2016; 17: 601-7.

22. Xing DG, Zhang DY, Wang ZF, Ding DL, Wang J, Wang YJ. Correlations of ANP genetic polymorphisms and serum levels with ischemic stroke risk: a meta-analysis. Genet Test Mol Biomarkers 2014; 18: 349-56.

23. Hakala T, Hedman A, Turpeinen A, et al. Prediction of atrial fibrillation after coronary artery bypass grafting by measuring atrial peptide levels and preoperative atrial dimensions. Eur J Cardiothorac Surg 2002; 22: 939-43.

24. Jideus L, Blomstrom P, Nilsson L, et al. Tachyarrhythmias and triggering factors for atrial fibrillation after coro- 
nary artery bypass operation. Ann Thorac Surg 2000; 69: 1064-9.

25. Loke I, Squire IB, Davies JE, Ng LL. Reference ranges for natriuretic peptides for diagnostic use are dependent on age, gender and heart rate. Eur J Heart Fail 2003; 5: 599-606.

26. Yoshihara F, Nishikimi T, Sasako Y, et al. Plasma atrial natriuretic peptide concentration inversely correlates with left atrial collagen volume fraction in patients with atrial fibrillation. J Am Coll Cardiol 2002; 39: 288-94.

27. Leone O, Boriani G, Chiappini B, et al. Amyloid deposition as a cause of atrial remodelling in persistent valvular atrial fibrillation. Eur Heart J 2004; 25: 1237-41.

28. Rossi A, Enriquez-Sarano M, Burnett JC Jr, Lerman A, Abel MD, Seward JB. Natriuretic peptide levels in atrial fibrillation: a prospective hormonal and Doppler-echocardiographic study. J Am Coll Cardiol 2000; 35: 1256-62.

29. Chello M, Mastroroberto P, Perticone F, et al. Plasma levels of atrial and brain natriuretic peptides as indicators of recovery of left ventricular systolic function after coronary artery bypass. Eur J Cardiothorac Surg 2001; 20: 140-6.

30. Wazni OM, Martin DO, Marrouche NF, et al. Plasma B-type natriuretic peptide levels predict postoperative atrial fibrillation in patients undergoing cardiac surgery. Circulation 2004; 110: 124-7.

31. Masson S, Wu JH, Simon C, et al. Circulating cardiac biomarkers and postoperative atrial fibrillation in the OPERA trial. Eur J Clin Invest 2015; 45: 170-8. 